\title{
26 \\ Performance Analysis of the Dual Cell Spacer in ATM Systems
}

\author{
Michael Ritter \\ University of Würzburg, Institute of Computer Science \\ Am Hubland, D - 97074 Würzburg, Germany \\ Tel: +49931888 5505, Fax: +499318884601 \\ e-mail: ritter@informatik.uni-wuerzburg.de
}

\begin{abstract}
In this paper we develop an analysis of the so-called Dual Cell Spacer. In contrast to a conventional spacer, which shapes a traffic stream only according to a given Peak Cell Rate, the Dual Cell Spacer takes into account the Peak Cell Rate as well as the Sustainable Cell Rate with the corresponding Burst Tolerance. The analysis is carried out in the discrete-time domain. Performance measures such as the cell rejection probability, the cell delay distribution and the cell inter-departure time distribution are derived. All results are of an exact nature. Numerical examples that compare the performance of the Dual Cell Spacer with that of a conventional spacer show a similar performance in terms of cell delay and cell loss for relatively small values of the Burst Tolerance. Using our analysis, both the Sustainable Cell Rate and the Burst Tolerance, which are used for traffic shaping in the Dual Cell Spacer, can be chosen adequately to achieve a given target cell rejection probability or cell delay, respectively, and to allow the network provider to obtain a high multiplexing gain.
\end{abstract}

\section{Keywords}

Cell Delay Distribution, Cell Inter-Departure Process, Discrete-Time Analysis, Performance Comparison, Source Traffic Descriptors, Traffic Shaping 


\section{Introduction}

In ATM networks, resource allocation is performed on the basis of a traffic contract which is negotiated between the network and the user. One part of this traffic contract consists of source traffic descriptors such as the Peak Cell Rate (PCR) and the Sustainable Cell Rate (SCR) of the connection. As long as the source is well behaving, i.e. the negotiated rates are not exceeded, the network commits to meet certain Grade of Service (GoS) parameters such as the Cell Loss Rate (CLR), the Cell Transfer Delay (CTD) or the Cell Delay Variation (CDV), which constitute the other part of the traffic contract.

A major problem is the selection of adequate source traffic descriptors which are used for Usage Parameter Control (UPC) at the User Network Interface (UNI) or the Inter Network Interface (INI). On the one hand, these traffic descriptors are difficult to choose because of the burstiness and the unpredictable bandwidth variations of certain traffic sources, e.g. video and LAN-to-LAN traffic. On the other hand, they must be chosen to be useful for Connection Admission Control (CAC) and resource allocation.

For sources without hard time constraints, i.e. delay insensitive applications, traffic shaping can be performed to avoid congestion which may be caused by clusters of cells belonging to one connection. An advantage of shaping is, that it not only limits the traffic volume but also reduces the burstiness of the traffic source considerably. This allows an easier choice of the source traffic descriptors.

The way a traffic shaper works is to delay certain cells of a given connection such that the cell inter-departure times of consecutive cells are never smaller than a given value (cf. $[3,7,16]$ ). This is done at the expense of introducing delay. In general, a maximum delay bound will be fixed in the traffic contract which leads to the discarding of a cell if the cell would have to be delayed longer than this amount of time. Each incoming cell is therefore either discarded or buffered in the shaper, and re-emitted so that the resulting output cell stream is conforming with the negotiated source traffic descriptors.

At connection setup, the declaration of the PCR is necessary. However, the SCR can be specified optionally $[1,10]$. This must be done in conjunction with a Burst Tolerance (BT), which limits the number of cells that can be transmitted at PCR. The declaration of the SCR may allow the network provider to utilize the network resources more efficiently.

Until now, only traffic shaping according to a given PCR has been considered in the literature, where performance measures such as cell loss, cell delay, and characteristics of the output process have been addressed for various traffic models, see e.g. $[2,4,5,9,11]$. Such a shaping facility is often called Cell Spacer. However, if a SCR is negotiated, a cell stream can be shaped to be conforming with both the PCR and the SCR specified in conjunction with the BT. The complexity of such a Dual Cell Spacer would only be slightly higher than that of the Conventional Spacer treated in the literature until now. Using a Dual Cell Spacer, the PCR and the SCR can be guaranteed for a given connection. This approach is used e.g. in [6] for the forming of virtual paths carrying delay sensitive connections. 
In the following we describe the operation mode of the Dual Cell Spacer. Its basic function is to enforce a minimum cell inter-departure time, aiming at the policing of the PCR. The input cell stream is therefore influenced in such a way, that the time between cells in the output stream is at least $T$, if $1 / T$ is the PCR. However, if a burst, which is emitted at PCR, is getting too large, then the Dual Cell Spacer has to throttle down the cell emission rate to be conforming with the negotiated SCR $1 / T_{s}$. The Maximum Burst Size (MBS) depends on the BT $\tau_{s}$. Thus, a cell is delayed as long as necessary to be conforming with both the PCR and the SCR.

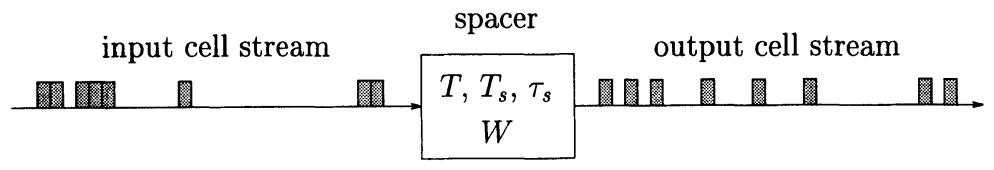

Abbildung 1: Basic Dual Cell Spacer model

Generally, a maximum CTD is declared in the traffic contract. Cells which have to be delayed longer than a certain delay bound $W$ are therefore rejected by the Dual Cell Spacer. This bound will be chosen according to the corresponding QoS parameter. In Figure 1, the basic model of the Dual Cell Spacer and related parameters are shown.

The remainder of this paper is organized as follows. In Section 2 we present an exact analysis of the Dual Cell Spacer. Performance measures addressed are the cell rejection probability, the cell inter-departure time distribution and the cell delay distribution. We assume the cell inter-arrival process to be a renewal process. Numerical examples are provided in Section 3 and the paper concludes with a brief summary in Section 4 .

\section{Analysis}

The basic idea behind the analysis is to introduce a spacer state denoted by the timedependent random variable $U(t)=(P(t), S(t))$. Depending on the spacer state $U(t)$ that a cell sees upon arrival, the cell is either delayed for a specific amount of time, is rejected if the delay bound $W$ would be exceeded, or departs immediately from the spacer. A similar approach has been used in [9] to analyze the Conventional Spacer. If the first component $P(t)$ is positive, it describes the amount of time a cell arriving at time $t$ has to be delayed to conform with the PCR. If $P(t)$ is negative at a cell arrival instant, then the cell can depart the spacer immediately, from the PCR shaping point of view. The second component $S(t)$ corresponds in a similar way to the SCR shaping part. The only difference is that the BT $\tau_{s}$ must be taken into account. Thus, if $S(t)$ is less than or equal to $\tau_{s}$, the cell can depart immediately. Otherwise, the cell has to be delayed by at least $S(t)-\tau_{s}$ to achieve conformance. This holds, of course, only from the SCR shaping point of view. 
As appropriate for ATM environments, the time is discretized into slots of cell duration. The following notation is used:

$P_{n}^{-} \quad P(t)$ just before the arrival instant of cell number $n$,

$P_{n}^{+} \quad P(t)$ just after the arrival instant of cell number $n$,

$S_{n}^{-} \quad S(t)$ just before the arrival instant of cell number $n$,

$S_{n}^{+} \quad S(t)$ just after the arrival instant of cell number $n$,

$A_{n}$ discrete random variable for the number of slots between the arrival instants of cells $n-1$ and $n$.

Accordingly, the discrete random variables

$$
U_{n}^{-}=\left(P_{n}^{-}, S_{n}^{-}\right) \quad \text { and } \quad U_{n}^{+}=\left(P_{n}^{+}, S_{n}^{+}\right)
$$

can be defined. The distributions of $U_{n}^{-}$and $U_{n}^{+}$are denoted by $u_{n}^{-}(i, j)$ and $u_{n}^{+}(i, j)$, where e.g. $u_{n}^{-}(i, j)$ is defined by $\operatorname{Pr}\left\{P_{n}^{-}=i \wedge S_{n}^{-}=j\right\}$. Furthermore, we denote the distribution of $A_{n}$ by $a(k)$, since the arrival process is assumed to be a renewal process. A sample evolution of the random variables defined above is depicted in Figure 2.

Starting with the arrival of cell $n-3$, we have $P_{n-3}^{-}=0$ and $S_{n-3}^{-}<\tau_{s}$. Thus, cell $n-3$ departs immediately from the spacer and the components $P(t)$ and $S(t)$ are increased by $T$ and $T_{s}$, respectively. Subsequently, they are decremented each slot. Just before the arrival of cell $n-2, S_{n-2}^{-}$is still smaller than $\tau_{s}$ but $P_{n-2}^{-}$is now larger than 0 . Therefore, cell $n-2$ must be delayed. Since $P_{n-2}^{-}<W$, cell $n-2$ is not rejected but delayed by $P_{n-2}^{-}$slots. At the arrival of cell $n-1$, we have $0<P_{n-1}^{-}<W$ and $\tau_{s}<S_{n-1}^{-}<\tau_{s}+W$. Cell $n-1$ is therefore not rejected but delayed by $P_{n-1}^{-}$, since $P_{n-1}^{-}>S_{n-1}^{-}-\tau_{s}$. The same holds for cell $n$, with the difference that $P_{n}^{-}<S_{n}^{-}-\tau_{s}$ and thus cell $n$ is delayed by $S_{n}^{-}-\tau_{s}$ slots. Just before cell $n+1$ arrives, $S_{n+1}^{-}>\tau_{s}+W$. Therefore, cell $n+1$ would have to be delayed longer than $W$ and is thus rejected and the system state is not increased. For the cells $n+2$ and $n+3$ we have the same situation as for cells $n-3$ and $n-2$, respectively. Finally, cell $n+4$ is rejected. The reason for this is that $P_{n+4}^{-}$is larger than the delay bound $W$.

In the following, we present an iterative algorithm to compute $u_{n}^{-}(i, j)$ and $u_{n}^{+}(i, j)$ if the cell arrival process follows a general distribution. The analysis is based on the algorithm for the computation of the system size distribution in the $G^{[X]} / D / 1 /-S$ queueing system presented in [15]. This algorithm has been used in $[8,12,14]$ to investigate UPC functions such as the Generic Cell Rate Algorithm (GCRA) and an extension to analyze twodimensional state processes has been presented in [13]. As outlined in the next three subsections, performance measures such as the cell rejection probability, the cell interdeparture time distribution, and the cell delay distribution can be derived easily using the limiting distribution $u^{-}(i, j)$. 


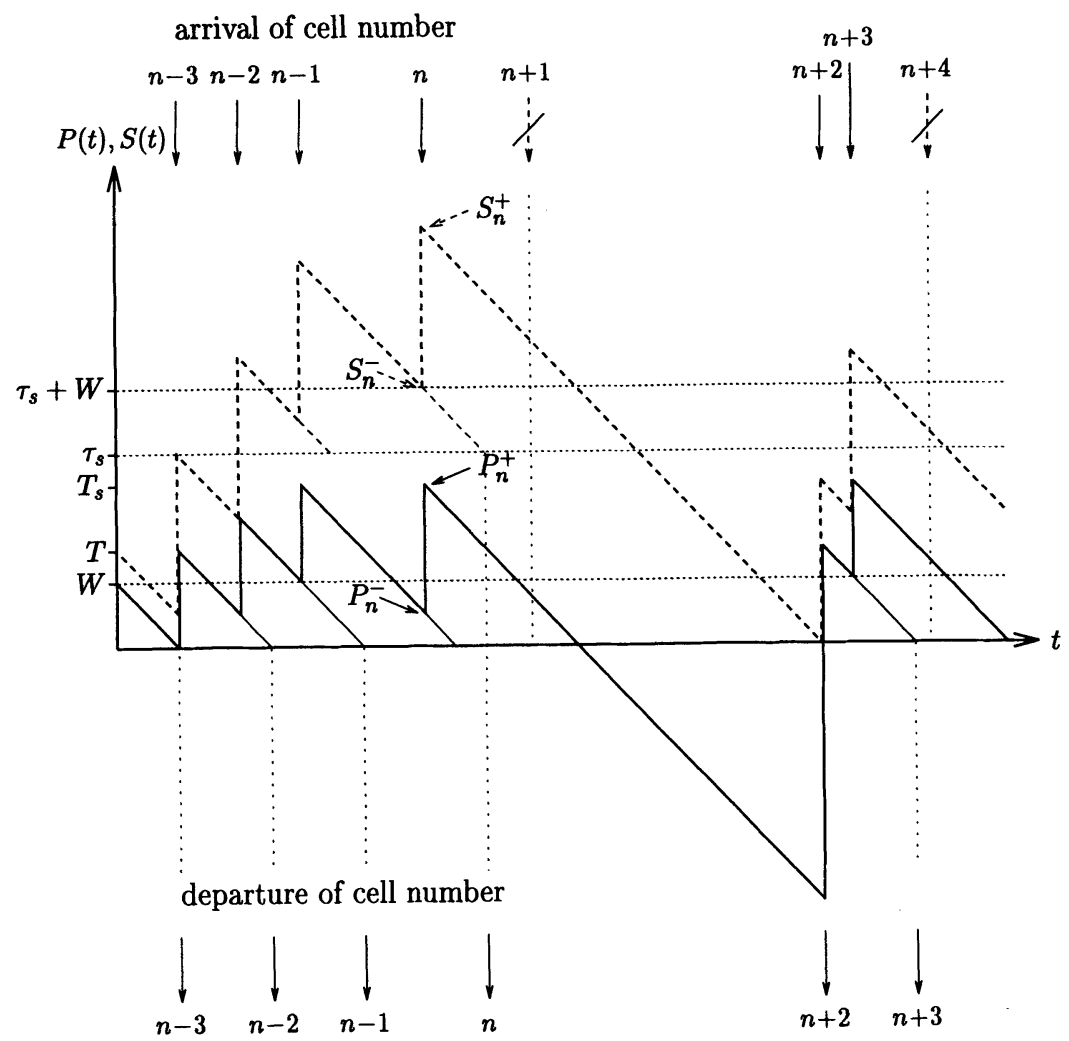

Abbildung 2: Sample path of the Dual Cell Spacer state process.

We start with the dependencies between the random variables $U_{n+1}^{-}=\left(P_{n+1}^{-}, S_{n+1}^{-}\right)$and $U_{n}^{+}=\left(P_{n}^{+}, S_{n}^{+}\right)$:

$$
\begin{aligned}
& P_{n+1}^{-}=P_{n}^{+}-A_{n+1}, \\
& S_{n+1}^{-}=S_{n}^{+}-A_{n+1} .
\end{aligned}
$$

These equations are driven by the decrease of $P(t)$ and $S(t)$ by one each slot. Based on the equations (2) and (3), the distribution $u_{n+1}^{-}(i, j)$ can be found as:

$$
u_{n+1}^{-}(i, j)=\sum_{k=-\infty}^{0} u_{n}^{+}(i-k, j-k) \cdot a(-k)
$$


The computation of $U_{n}^{+}=\left(P_{n}^{+}, S_{n}^{+}\right)$from $U_{n}^{-}=\left(P_{n}^{-}, S_{n}^{-}\right)$is more complicated. For $P_{n}^{+}$ and $S_{n}^{+}$we get the following:

$$
\begin{aligned}
& P_{n}^{+}=\left\{\begin{array}{lll}
T & : \quad P_{n}^{-} \leq 0 \quad \wedge \quad S_{n}^{-} \leq \tau_{s}+W \\
P_{n}^{-}+T & : & 0<P_{n}^{-} \leq W \quad \wedge \quad S_{n}^{-} \leq \tau_{s}+W \\
P_{n}^{-} & : & P_{n}^{-}>W \quad \vee \quad S_{n}^{-}>\tau_{s}+W
\end{array}\right. \\
& S_{n}^{+}=\left\{\begin{array}{lll}
T_{s} & : & S_{n}^{-} \leq 0 \wedge P_{n}^{-} \leq W \\
S_{n}^{-}+T_{s} & : & 0<S_{n}^{-} \leq \tau_{s}+W \quad \wedge \quad P_{n}^{-} \leq W \\
S_{n}^{-} & : & S_{n}^{-}>\tau_{s}+W \quad \vee \quad P_{n}^{-}>W
\end{array}\right.
\end{aligned}
$$

First, let us focus on $P(t)$, i.e. equation (5). If $P_{n}^{-} \leq 0$ and cell $n$ is not rejected due to a delay longer than $W$ resulting from SCR shaping, $P_{n}^{+}$is set to $T$ since the time interval until the emission of the next cell should be at least $T$. This corresponds to the first case in equation (5). For a $P_{n}^{-}$larger than 0 , the first component of the spacer state is computed by $P_{n}^{+}=P_{n}^{-}+T$, if the delay according to the SCR shaping is smaller than $W$. The third case reflects the arrival of a cell which would have to be delayed longer than $W$ and thus the spacer state remains unchanged. Analogously, equation (6) describes the computation of the second component $S(t)$.

To derive the equations for the corresponding distributions, we have to distinguish the four cases illustrated in Figure 3. Regions with different shadings represent different ways of calculating $u_{n}^{+}(i, j)$ due to acceptance or rejection of cell $n$. Dashed lines exclude the points from the delimited regions, solid lines include them. For sake of clarity, we decomposed the transition into two steps.

The first step reflects a shifting operation which truncates the negative part of the spacer state space where arriving cells are not rejected. Therefore, the probabilities of states $(i, j)$, where $i$ or $j$ are negative, are shifted to those states where $i$ or $j$ are replaced by zero if negative. This transition can be expressed by:

$$
u_{n}^{*}(i, j)=\pi_{*}\left(u_{n}^{-}(i, j)\right)
$$

with the operator $\pi_{*}(\cdot)$ defined as:

$$
\pi_{*}\left(u_{n}^{-}(i, j)\right)=\left\{\begin{array}{ll}
0 & : i<0 \wedge j \leq \tau_{s}+W \\
0 & : 0 \leq i \leq W \wedge j<0 \\
\sum_{i^{\prime}=-\infty}^{0} u_{n}^{-}\left(i^{\prime}, j\right) & : i=0 \wedge 0<j \leq \tau_{s}+W \\
\sum_{i^{\prime}=-\infty}^{0} \sum_{j^{\prime}=-\infty}^{0} u_{n}^{-}\left(i^{\prime}, j^{\prime}\right) & : i=0 \wedge j=0 \\
\sum_{j^{\prime}=-\infty}^{0} u_{n}^{-}\left(i, j^{\prime}\right) & : 0<i \leq W \wedge j=0 \\
u_{n}^{-}(i, j) & : \text { otherwise }
\end{array} .\right.
$$




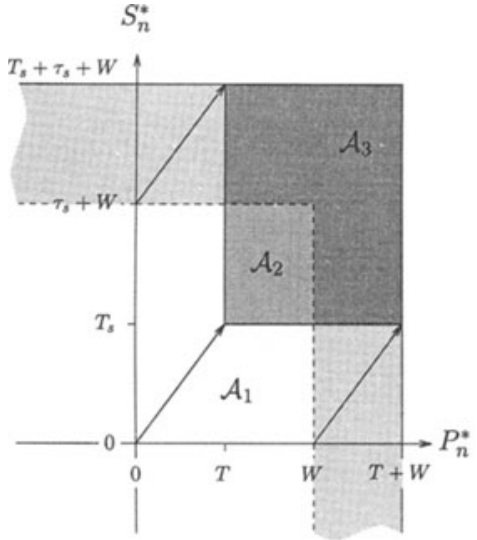

Case 1: $W \geq T \wedge \tau_{s}+W \geq T_{s}$

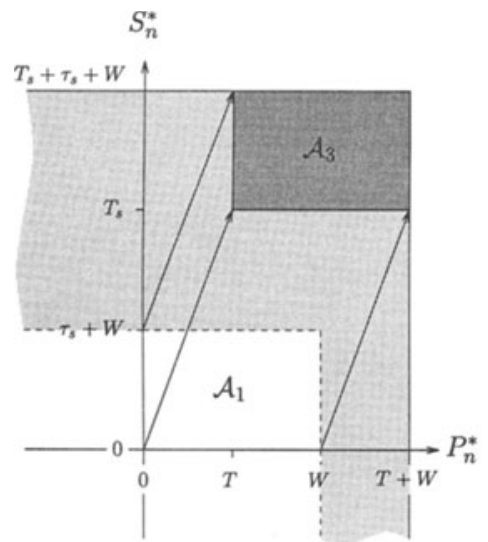

Case 3: $W \geq T \wedge \tau_{s}+W<T_{s}$

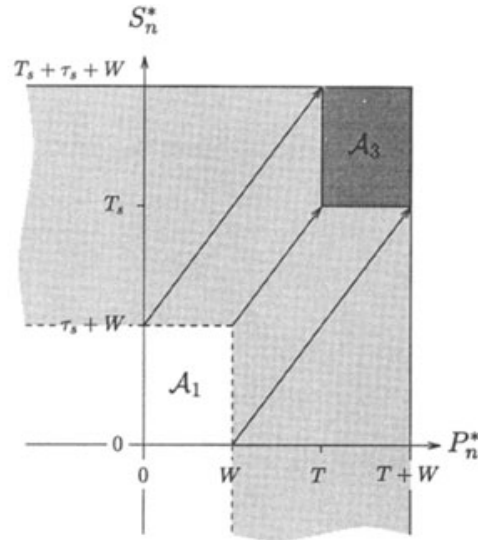

Case 2: $W<T \wedge \tau_{s}+W<T_{s}$

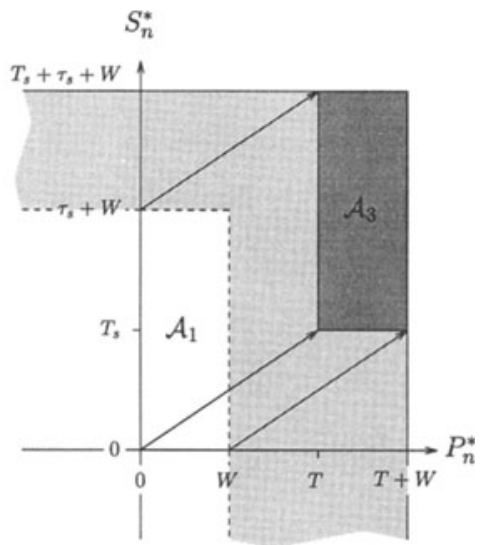

Case 4: $W<T \wedge \tau_{s}+W \geq T_{s}$

Abbildung 3: Transition regions for $u_{n}^{*}(i)$ to $u_{n}^{+}(i)$

In the second step, i.e. the transition from $u_{n}^{*}(i, j)$ to $u_{n}^{+}(i, j)$, the resulting region in which cells are not rejected is shifted by the vector $\left(T, T_{s}\right)$. This leads to the following equation:

$$
u_{n}^{+}(i, j)=\left\{\begin{array}{lll}
0 & :(i, j) \in \mathcal{A}_{1} \\
u_{n}^{*}\left(i-T, j-T_{s}\right) & :(i, j) \in \mathcal{A}_{2} \\
u_{n}^{*}\left(i-T, j-T_{s}\right)+u_{n}^{*}(i, j) & : & (i, j) \in \mathcal{A}_{3} \\
u_{n}^{*}(i, j) & : \text { otherwise }
\end{array}\right.
$$


For case 1 , the regions $\mathcal{A}_{1}, \mathcal{A}_{2}$ and $\mathcal{A}_{3}$ (cf. Figure 3 ) are given by:

$$
\begin{aligned}
\mathcal{A}_{1}=\{(i, j) \mid & \left(0 \leq i \leq W \wedge \quad 0 \leq j<T_{s}\right) \vee \\
& \left.\left(0 \leq i<T \wedge \quad 0 \leq j \leq \tau_{s}+W\right)\right\} \\
\mathcal{A}_{2}=\{(i, j) \mid & \left.\left(T \leq i \leq W \wedge T_{s} \leq j \leq \tau_{s}+W\right)\right\}, \\
\mathcal{A}_{3}=\{(i, j) \mid & \left(W<i \leq T+W \wedge T_{s} \leq j \leq T_{s}+\tau_{s}+W\right) \quad \vee \\
& \left.\left(T \leq i \leq T+W \wedge \tau_{s}+W<j \leq T_{s}+\tau_{s}+W\right)\right\} .
\end{aligned}
$$

For all other cases depicted in Figure 3, these regions are defined as follows:

$$
\begin{aligned}
& \mathcal{A}_{1}=\left\{(i, j) \mid\left(0 \leq i \leq W \wedge 0 \leq j \leq \tau_{s}+W\right)\right\} \\
& \mathcal{A}_{2}=\emptyset \\
& \mathcal{A}_{3}=\left\{(i, j) \mid\left(T \leq i \leq T+W \quad \wedge \quad T_{s} \leq j \leq T_{s}+\tau_{s}+W\right)\right\} .
\end{aligned}
$$

Using the equations presented above, the limiting distribution of the spacer state distribution $u^{-}(i, j)$ can iteratively be obtained:

$$
u^{-}(i, j)=\lim _{n \rightarrow \infty} u_{n}^{-}(i, j)
$$

This forms the basis for the derivation of the performance measures outlined in the next three subsections.

\subsection{Cell rejection analysis}

The cell rejection probability $p_{r}$, i.e. the probability that an arriving cell has to be delayed longer than the delay bound $W$, can be found as

$$
p_{r}=1-\sum_{i=-\infty}^{W} \sum_{j=-\infty}^{\tau_{s}+W} u^{-}(i, j)
$$

The rejection probability $p_{r}$ is given by the probability of the area of the spacer state space where an arriving cell has to be delayed longer than $W$ either because of PCR shaping or because of SCR shaping. 


\subsection{Cell inter-departure time distribution}

In this subsection we focus on the cell inter-departure time distribution of the Dual Cell Spacer. This distribution can be used e.g. to model the input traffic of an ATM multiplexer. The following derivation of the output process is performed in conjunction with a renewal assumption.

The probability $d(k)$ to observe a time interval of $k$ slots between two cells departing the spacer consecutively can be given by:

$$
d(k)=\frac{1}{1-p_{r}} \cdot \sum_{(i, j) \in \mathcal{B}_{k}} u^{-}(i, j)
$$

In this context, the set $\mathcal{B}_{k}$ contains those states $(i, j)$ where the departure of the previous accepted cell has occurred or will occur $k$ slots before the departure of the cell which is currently arriving.

Due to the PCR shaping, we have a minimum cell inter-departure interval of $T$ slots and thus for $k<T$ :

$$
\mathcal{B}_{k}=\emptyset
$$

To derive the sets $\mathcal{B}_{k}$ for $k \geq T$, we have to distinguish three regions of the spacer state space, which are depicted in Figure 4 with different shading intensities.

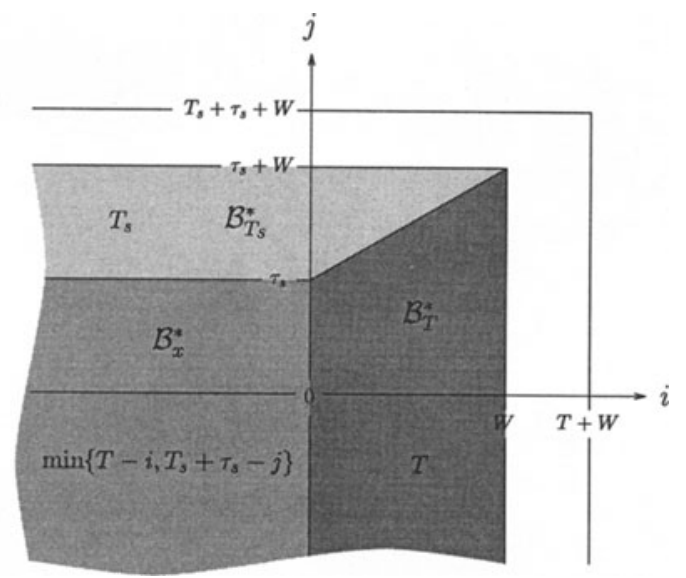

Abbildung 4: Regions for calculating the cell inter-departure time distribution 
If an arriving cell does not need to be delayed, then the cell inter-departure interval for the actual spacer state $(i, j)$ is determined by $\min \left\{T-i, T_{s}+\tau_{s}-j\right\}$. This is relevant if $(i, j)$ is located in the region $\mathcal{B}_{x}^{*}$ (cf. Figure 4). If $i \leq 0$, then the last departure has occurred $T-i$ slots before. For $j \leq \tau_{s}$ the last departure instant has occurred $T_{s}+\tau_{s}-j$ time slots before. Since PCR and SCR shaping are performed independently of each other, the cell inter-departure interval is, in this case, equal to the minimum of the two values above.

If the spacer state upon cell arrival is located in the dark shaded region $\mathcal{B}_{T}^{*}$, the cell interdeparture interval will be $T$, since there is no further delay required due to SCR shaping. For the spacer states in the light shaded region $\mathcal{B}_{T_{s}}^{*}$, an arriving cell has to be delayed due to SCR shaping. Thus the cell inter-departure interval is equal to $T_{s}$.

Taking these properties into account, the sets $\mathcal{B}_{T}$ and $\mathcal{B}_{T_{s}}$ are given by:

$$
\begin{aligned}
\mathcal{B}_{T}=\{(i, j) \mid & \left(0<i \leq W \wedge-\infty<j \leq i+\tau_{s}\right) \quad \vee \\
& \left.\left(\min \left\{T-i, T_{s}+\tau_{s}-j\right\}=T\right)\right\}
\end{aligned}
$$

and

$$
\begin{aligned}
\mathcal{B}_{T_{s}}=\{(i, j) \mid & \left(\tau_{s}<j \leq \tau_{s}+W \wedge-\infty<i<j-\tau_{s}\right) \quad \vee \\
& \left.\left(\min \left\{T-i, T_{s}+\tau_{s}-j\right\}=T_{s}\right)\right\} .
\end{aligned}
$$

For all other values of $k, \mathcal{B}_{k}$ contains the following states:

$$
\mathcal{B}_{k}=\left\{(i, j) \mid \min \left\{T-i, T_{s}+\tau_{s}-j\right\}=k\right\} .
$$

\subsection{Cell delay distribution}

The performance measure addressed in this subsection is the cell delay distribution. This measure allows e.g. to investigate the additional delay introduced by SCR shaping compared to that of a Conventional Spacer.

Since the delay for cells that arrive at time instants where one or both components of the spacer state are negative is the same as for time instants where the corresponding component is equal to 0 , we can make use of the shifting operation given in equation (8):

$$
u^{*}(i, j)=\pi_{*}\left(u^{-}(i, j)\right)
$$


Defining sets $\mathcal{C}_{k}$ which contain those states $(i, j)$ where an arriving cell is delayed by $k$ slots, we can compute the cell delay distribution $w(k)$ for $k=0, \ldots, W$ by the following equation:

$$
w(k)=\frac{1}{1-p_{r}} \cdot \sum_{(i, j) \in \mathcal{C}_{k}} u^{*}(i, j)
$$

A cell is delayed by $k$ slots, if the delay due to PCR shaping is equal to $k$ slots and the required delay due to SCR is shorter or vice versa. Thus, for $0 \leq k \leq W$ the sets $\mathcal{C}_{k}$ are given by:

$$
\mathcal{C}_{k}=\left\{(i, j) \mid\left(i=k \wedge i>j-\tau_{s}\right) \quad \vee \quad\left(j=k+\tau_{s} \wedge i \leq j-\tau_{s}\right)\right\} .
$$

Because of the maximum delay bound $W$, the sets $\mathcal{C}_{k}$ are empty for $k>W$. The probability $p_{w}$ that an arriving cell has to be delayed can be computed by:

$$
p_{w}=1-w(0)
$$

\section{Numerical results}

To compare the performance of the Dual Cell Spacer with that of the Conventional Spacer, we present a number of numerical results. In Figure 5, cell delay distributions are drawn for several choices of $\tau_{s}$. The inter-arrival process used is a negative-binomial distribution which allows varying the mean $E_{A}$ and the coefficient of variation $c_{A}$ almost independently of each other. Of course, $E_{A} \cdot c_{A}^{2}>1$ must be fulfilled. We use a mean of $E_{A}=10.0$ slots and the coefficient of variation is set to $c_{A}=1.0$. The spacer parameters are $T=5$, $T_{s}=8$ and $\tau_{s}$ is varied from 0 to 150 . A maximum delay of $W=200$ slots is tolerated.

As can be observed in Figure 5, the cell delay distribution is strongly dependent on the choice of $\tau_{s}$. If $\tau_{s}$ increases, the cell delay distribution rapidly approaches that of the Conventional Spacer. For $\tau_{s}=\infty$, both are the same, since for a BT tending to infinity the SCR shaping has no effect on the delay of the cells. In our case, the distributions for $\tau_{s}=\infty$ and $\tau_{s}=150$ can not be distinguished.

If we look at Figure 6 where the coefficient of variation is now set to $c_{A}=2.0$, a much slower approach can be observed, i.e. $\tau_{s}$ must be chosen large to obtain a cell delay distribution close to that of a conventional spacer. Each of the distribution functions in the Figures 5 and 6 shows small steps for short delays, as typical for $G I / D / 1$ queueing systems.

In Figure 7, the mean cell delay is plotted over $\tau_{s}$ for various choices of $c_{A}$. This figure shows the asymptotic behavior more clearly. The smaller the value of $c_{A}$, the faster the mean cell delay approaches a limiting value. 


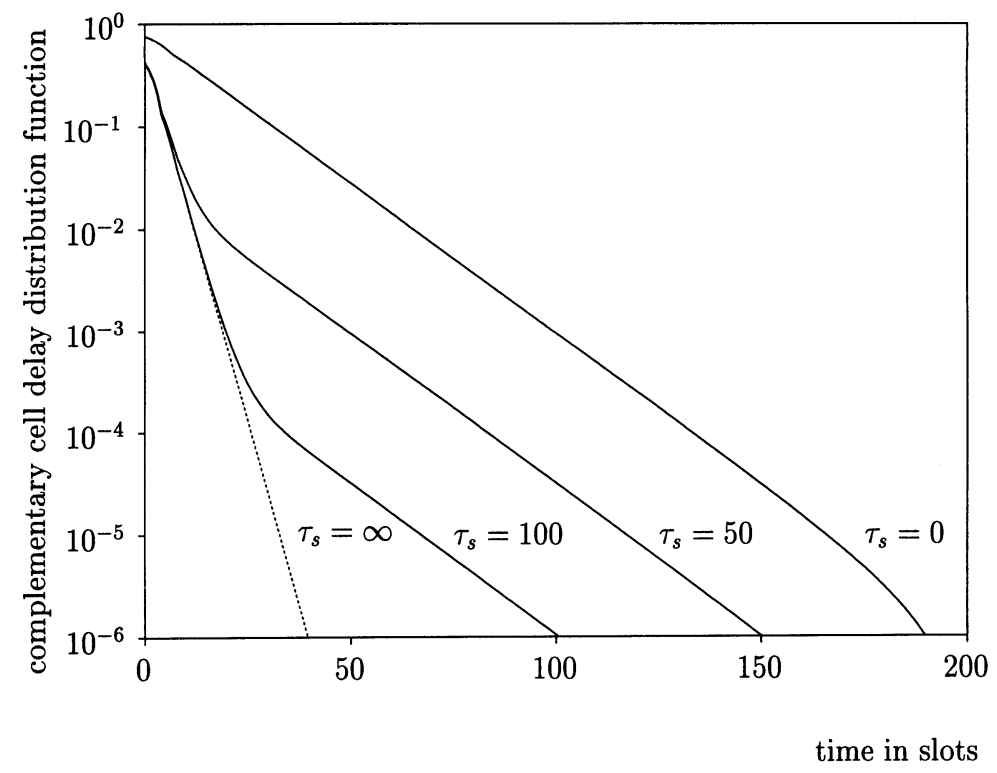

Abbildung 5: Influence of the BT $\tau_{s}$ on the cell delay distribution $\left(c_{A}=1.0\right)$

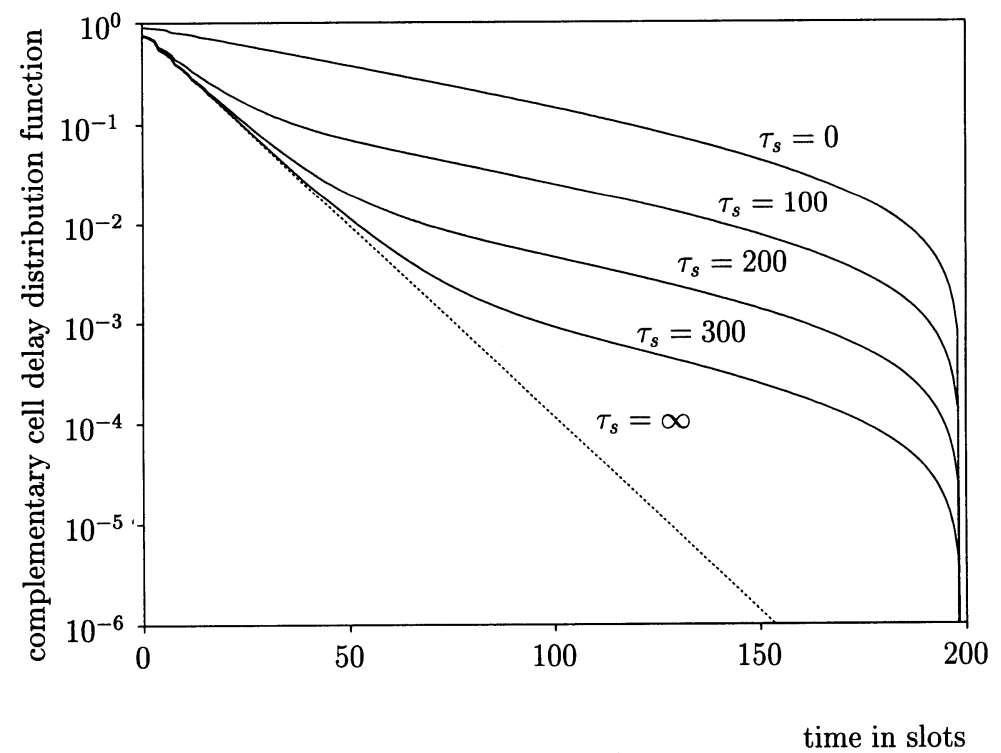

Abbildung 6: Influence of the BT $\tau_{s}$ on the cell delay distribution $\left(c_{A}=2.0\right)$ 
Thus, the BT should be dimensioned small in order to preserve the supplementary property of the dual mechanism, i.e. reducing the burstiness of the traffic stream. This depends, however, on the choice of the SCR $1 / T_{s}$. If the SCR value is close to the Average Cell Rate, SCR shaping plays a dominant role even for large values of the BT.

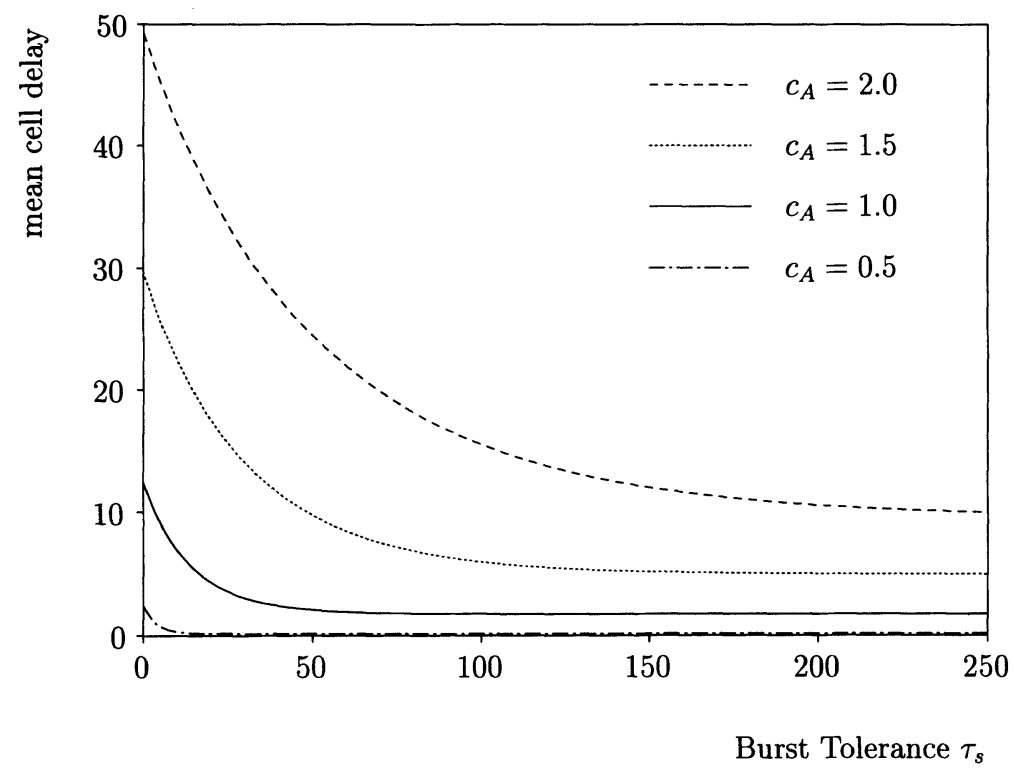

Abbildung 7: Asymptotic behavior of the mean cell delay

Another interesting performance measure is the coefficient of variation $c_{W}$ of the delay introduced by the Dual Cell Spacer. Curves for different choices of $c_{A}$ are depicted in Figure 8 as a function of $\tau_{s}$. As expected, $c_{W}$ also approaches a limiting value if the BT is increased. This is due to the approach of the cell delay distribution against a limiting distribution as shown in the Figures 5 and 6.

For small values of $\tau_{s}$, the coefficient of variation $c_{W}$ first increases and then decreases against a limiting value. The initial increase is more distinctive if $c_{A}$ is small. The later decrease of the cell delay coefficient of variation is due to the minor influence of SCR shaping for large values of $\tau_{s}$. If $c_{A}$ is larger, the curves are located closer to each other.

The next measure we focus on is the cell inter-departure time distribution of the Dual Cell Spacer. In Figure 9, we use the same inter-arrival process as before with a coefficient of variation $c_{A}=2.0$. For $\tau_{s}=0$, i.e. no bursts are allowed, the cell inter-departure time distribution is equal to that of a Conventional Spacer with minimum inter-cell distance $T_{s}$. 


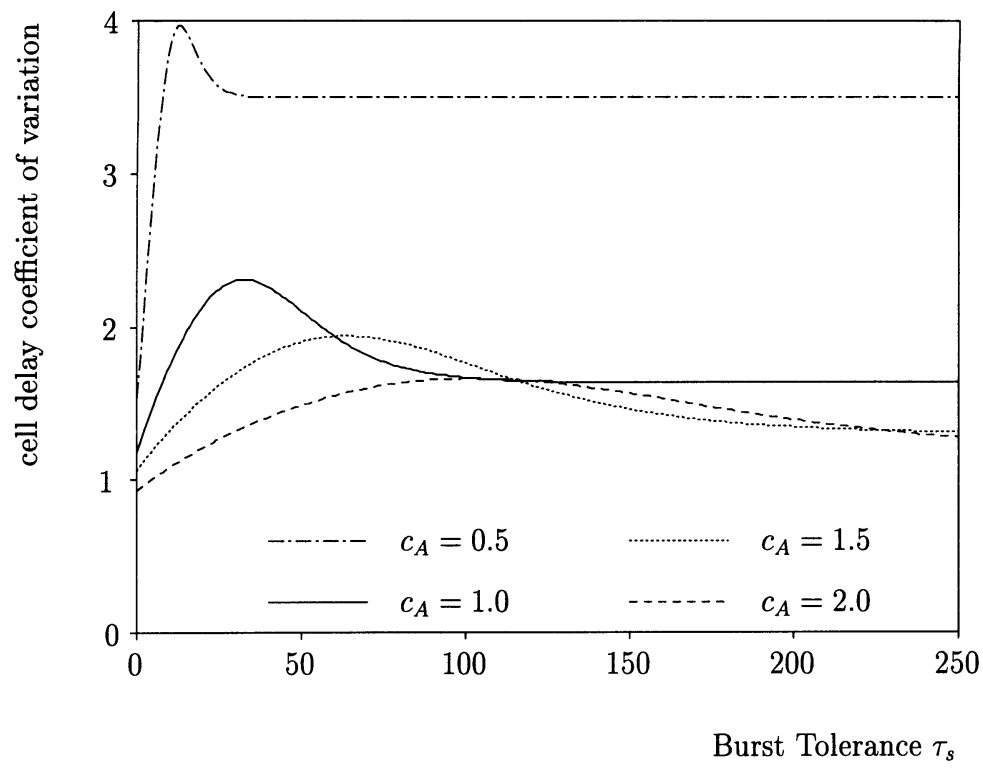

Abbildung 8: Asymptotic behavior of the cell delay coefficient of variation $c_{W}$

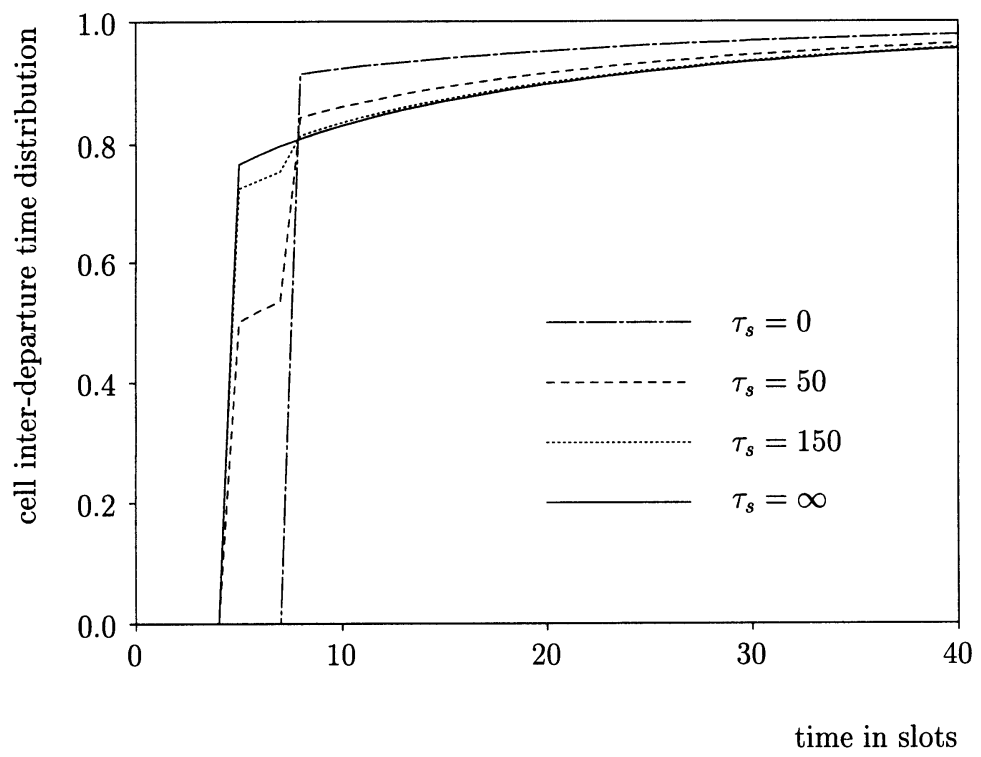

Abbildung 9: Influence of $\tau_{s}$ on the cell inter-departure time distribution 
If $\tau_{s}$ is increased, the cell inter-departure time distribution also approaches a limiting distribution. As in case of the cell delay distribution, this limiting distribution is equal to the cell inter-departure time distribution of the Conventional Spacer with a minimum inter-cell distance of $T$.

For all other values of $\tau_{s}$, two steps can be observed. These steps correspond to the PCR and SCR shaping and are therefore located at $i=5$ and $i_{s}=8$. Note that $T=5$ and $T_{s}=8$. The step due to SCR shaping gets smaller if $\tau_{s}$ increases or $c_{A}$ decreases.

From the network point of view, a small value of the BT may allow a higher utilization of the network resources since the traffic stream is smoother. However, the cell rejection probability and the cell delay will be increased. Using our analysis, the tuple $\left(T_{s}, \tau_{s}\right)$ can be chosen to achieve a given target cell rejection probability or cell delay, respectively, and to allow the network provider to obtain a high multiplexing gain at the same time.

In general, a user's decision whether to shape traffic only according to a PCR or according to both PCR and SCR strongly depends on the way the tariffing is carried out. Consider for example a user application which allows a mean cell delay of at most 20 cell times and assume that the cell arrival process follows a negative-binomial distribution with $E_{A}=10.0$ slots and $c_{A}=2.0$.

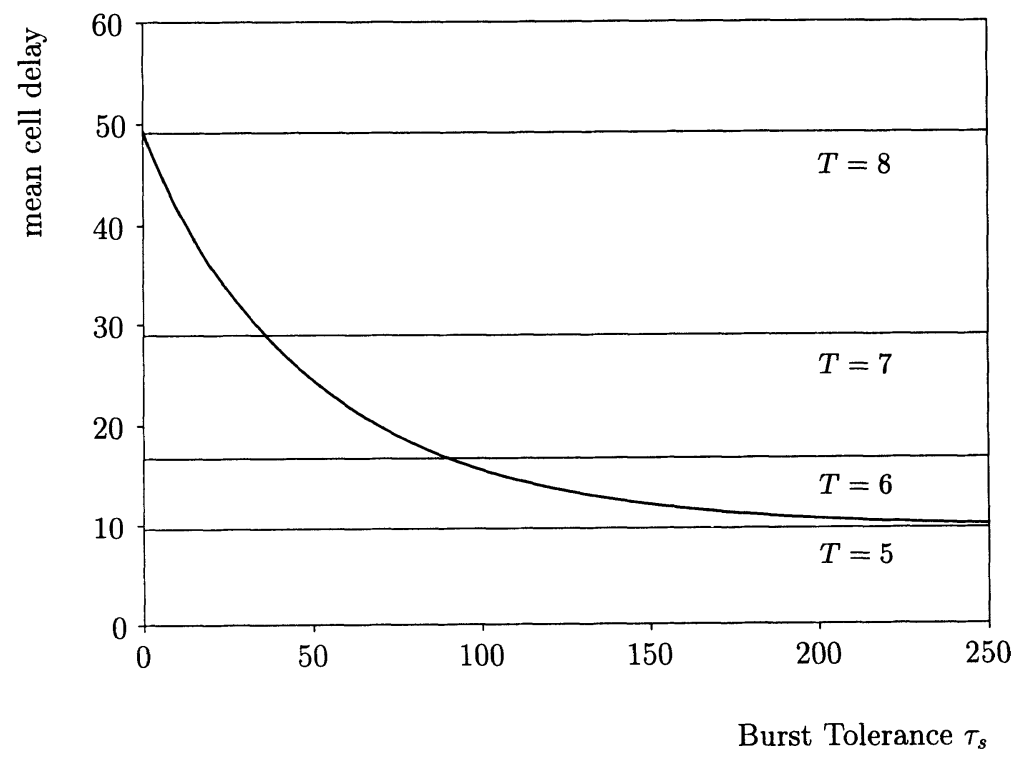

Abbildung 10: Dual Cell Spacer vs. Conventional Spacer 
In Figure 10 we have drawn the mean cell delay for a Dual Cell Spacer with $T=5, T_{s}=8$ and $W=200$ as a function of the BT. We also plotted horizontal lines for the mean cell delay for a Conventional Spacer with $T=5,6,7,8$ and the same maximum delay bound $W$.

The user has in principle two possibilities to shape traffic in order to achieve a mean cell delay of less than 20 cell times:

1. use a Conventional Spacer and specify a PCR with $T=6$,

2. use a Dual Cell Spacer and specify e.g. a PCR with $T=5$ and additionally a SCR of $T=8$ with a BT $\tau_{s} \approx 80$.

Of course, the user's choice will depend on the tariffing policy, since the user will choose the cheaper alternative for the connection.

\section{Conclusion}

In this paper we presented a discrete-time analysis of the Dual Cell Spacer which shapes an ATM input traffic stream to be conforming with a given Peak Cell Rate and a Sustainable Cell Rate in conjunction with the Burst Tolerance. The input traffic is assumed to be a renewal process and a maximum delay bound for the spacer is introduced. Using the limiting distribution of the two-dimensional system state distribution, performance measures such as the cell rejection probability, the cell inter-departure time distribution and the cell delay distribution have been derived in closed form. All results are of an exact nature. Numerical examples are given to show the performance of the Dual Cell Spacer for different traffic conditions. Furthermore, the results have been compared with those of a Conventional Spacer, i.e. a spacer which shapes the traffic only according to a Peak Cell Rate.

From the numerical examples we can conclude that already for small values of the Burst Tolerance, the Dual Cell Spacer achieves performance close to that of the Conventional Spacer, if the Sustainable Cell Rate is chosen adequately. The reason for this is the fast approach of the cell delay and the cell inter-departure time distribution against their limiting distributions. Thus, the supplementary properties introduced by the dual mechanism are lost already for relatively small values of the Burst Tolerance.

If an additional delay can be accepted, the Burst Tolerance can however be chosen quite small resulting in a traffic stream which is very smooth and therefore favorable from the network point of view. Since the network provider needs to allocate fewer resources when the negotiated Burst Tolerance is small, a small Burst Tolerance in conjunction with a reasonable choice of the Sustainable Cell Rate may also be profitable for the user from the tariffing point of view. 


\section{Acknowledgement}

The author appreciates the support of the Research Institute of the Deutsche Telekom AG (FZ Darmstadt).

\section{Literatur}

[1] ATM Forum. ATM User-Network Interface Specification. Version 3.1, September 1994.

[2] F. Bernabei, L. Gratta, M. Listanti, A. Sarghini. Analysis of ON-OFF Source Shaping for ATM Multiplexing. IEEE INFOCOM 1993, pp. 1330-1336.

[3] P. Boyer, F. Guillemin, M. Servel, J.-P. Coudreuse. Spacing Cells Protects and Enhances Utilization of ATM Network Links. IEEE Network, Vol. 6, No. 5, September 1992, pp. 38-49.

[4] F.M. Brochin. A Cell Spacing Device for Congestion Control in ATM Networks. Performance Evaluation, Vol. 16, No. 1, 1992, pp. 107-127.

[5] A.I. Elwalid, D. Mitra. Analysis and Design of Rate-Based Congestion Control of High Speed Networks, I: Stochastic Fluid Models, Access Regulation. Queueing Systems 9, 1991, pp. 29-64.

[6] J. García, M. Ritter. Determination of Traffic Descriptors for VPs Carrying DelaySensitive Traffic. submitted to INFOCOM 1996.

[7] F. Guillemin, P. Boyer, L. Romoeuf. The Spacer-Controller: Architecture and First Assessments. Broadband Communications, Portugal, January 1992, pp. 313-323.

[8] F. Hübner. Dimensioning of a Peak Cell Rate Monitor Algorithm Using DiscreteTime Analysis. Proceedings of ITC-14, Antibes, France, June 1994, pp. 1415-1424.

[9] F. Hübner, P. Tran-Gia. A Discrete-Time Analysis of Cell Spacing in ATM Systems. to appear in Telecommunication Systems.

[10] ITU-T Study Group 13, Draft Recommendation I.371. Traffic Control and Congestion Control in B-ISDN. March 1994.

[11] L.K. Reiss, L.F. Merakos. Shaping of Virtual Path Traffic for ATM B-ISDN. IEEE INFOCOM 1993, pp. 168-175.

[12] M. Ritter, P. Tran-Gia. Performance Analysis of Cell Rate Monitoring Mechanisms in ATM Systems. International Conference on Local and Metropolitan Communication Systems, Kyoto, December, 1994. 
[13] M. Ritter, S. Kornprobst, F. Hübner. Performance Comparison of Source Policing Architectures in ATM Systems. University of Würzburg, Institute of Computer Science, Research Report Series, Report No. 81, November 1994.

[14] O. Rose, M. Ritter. MPEG-Video Sources in ATM-Systems - A new approach for the dimensioning of policing functions. International Conference on Local and Metropolitan Communication Systems, Kyoto, December, 1994.

[15] P. Tran-Gia, H. Ahmadi. Analysis of a Discrete-Time $G^{[X]} / D / 1-S$ Queueing System with Applications in Packet-Switching Systems. IEEE INFOCOM 1988, pp. 861-870.

[16] E. Wallmeier, T. Worster. The Spacing Policer, an Algorithm for Efficient Peak Bit Rate Control in ATM Networks. ISS 14, October 1992, paper A5.5. 